\title{
Invasions, subversions, contaminations
}

De quelques figures et lieux contemporains d'un Autre exceptionnel Invasions, Subversions, Infections. Some Comtemporaries Figures and Places of an Exceptional Other

\section{Marc Bernardot}

\section{(2) OpenEdition}

\section{Journals}

\section{Édition électronique}

URL : http://journals.openedition.org/conflits/18237

DOI : $10.4000 /$ conflits. 18237

ISSN : $1777-5345$

\section{Éditeur :}

CCLS - Centre d'études sur les conflits lilberté et sécurité, L'Harmattan

Édition imprimée

Date de publication : 31 décembre 2011

Pagination : 45-62

ISBN : 978-2-296-96617-8

ISSN : $1157-996 X$

\section{Référence électronique}

Marc Bernardot, «Invasions, subversions, contaminations », Cultures \& Conflits [En ligne], 84 | Hiver 2011, mis en ligne le 15 mars 2013, consulté le 30 mars 2021. URL : http://journals.openedition.org/ conflits/18237 ; DOI : https://doi.org/10.4000/conflits.18237 


\section{Invasions, subversions, contaminations De quelques figures et lieux contemporains $d^{\prime}$ 'un Autre exceptionnel 1}

\section{Marc BERNARDOT}

Marc Bernardot est professeur de sociologie à l'université du Havre. Il est membre du laboratoire CIRTAI, UMR IDEES et participe à l'équipe éditoriale du résean TERRA. Spécialiste de sociologie historique de l'État et de l'espace, il travaille actuellement sur les politiques antimigratoires. Il a récemment publié "Qui sont les indésirables? La production des catégories du refoulement", in Jaeger M. (ed.), Usagers ou citoyens ? De l'usage des catégories en action sociale et médicosociale, Dunod, 2011, pp. 101-114 et " $A$ la recherche des sans trace : cultures, espaces et citoyennetés ", in Galinon-Mélénec B. (ed.), L'Homme-trace, Presses du CNRS, 2011, pp. 331-347.

L e maintien et la consolidation des frontières étatiques procèdent à la fois de dispositifs matériels, de discours experts et de manipulations symboliques notamment en ce qui concerne l'élaboration des risques contre le bien public et la sécurité nationale. Cette combinaison de pratiques et de discours se matérialise tout particulièrement dans l'espace du camp d'internement incarnant l'expression de la puissance souveraine et fonctionnant comme une enclave et une frontière mobiles, modulables et déplaçables selon les menaces désignées par l'État ${ }^{2}$. Durant le Haut moyen âge les peurs et les signes annon-

1. J'ai bénéficié des conseils et des recommandations bibliographiques de A. Lemarchand, A. L. Stoler, H. Thomas et J. Valluy pour la rédaction de cet article. Qu'ils en soient ici remerciés, de même que les relecteurs anonymes de Cultures $\mathcal{E}$ Conflits.

2. Je propose de donner une définition extensive du camp. Il s'agit d'un type d'enfermement spécifique de la modernité composé à partir d'objets élémentaires - la baraque, le barbelé, le chemin de garde et le mirador, et qui a pour finalité le regroupement arbitraire d'individus en dehors du système pénitentiaire pour les tenir à l'écart, les forcer à travailler ou les punir. Mais plus encore que d'autres appareils répressifs comme la prison, les camps présentent une grande variété de formes et une propension à être adaptés à des missions différentes. C’est pourquoi j'ajoute à cette première définition empirique une seconde dimension. Il existe à la fois ce que l'on pourrait appeler une «forme-camp » que l'on retrouve bien au-delà du plan de conception militaire, une logique et une pensée (camp thinking) du camp marquées par l'hybridation des fonctions, l'indétermination des frontières entre légalité et illégalité et une partition asymétrique du monde. À ce sujet voir mon ouvrage Camps d'étrangers, Éditions du Croquant, Bellecombe-en-Bauges, 2008. Se reporter aussi à Gilroy P., Between Camps. 
ciateurs de catastrophes étaient interprétés conjointement et se renforçaient mutuellement ${ }^{3}$. Il en est de même de nos jours autour de trois menaces à la fois anciennes 4 et réamorcées dans le cadre globalisé de la postmodernité dominée par la notion de risque et une nouvelle culture de la peur : l'invasion, la subversion et la contamination. Distinctes, elles partagent néanmoins des points communs d'une part en ce qu'elles sont toutes trois constituées en tant que mettant en cause la survie d'unités politiques et ethniques et d'autre part qu'elles sont construites comme agissant essentiellement de manière invisible. Elles sont devenues des grilles de lecture des migrations depuis un siècle lorsque les mobilités collectives ont pris une dimension centrale dans les politiques intérieures et internationales. Ces notions circulent entre les discours scientifiques, médiatiques, institutionnels, artistiques ou populaires. Elles présentent des caractères à la fois potentiel, secret, invisible et global, et elles correspondent avec des notions floues et extensives, émergentes au XIX e siècle, telles que l'ordre public, la sécurité intérieure, l'insécurité ou la situation exceptionnelle. Elles sont tout aussi difficiles à définir ou à avérer précisément mais elles tirent leur force de cette imprécision 5 . L'évocation et la manipulation de ces sources de terreurs collectives participent de la désignation de groupes comme étrangers et minoritaires et de l'élaboration de contretypes permettant de délimiter négativement les contours des nations. Ces menaces fantômes trouvent à s'incarner dans tout individu ou groupe perçu comme étranger.

L'application de la loi martiale, à l'origine de la théorie des circonstances exceptionnelles ${ }^{6}$, passe par la construction de groupes humains en tant que « menaces » virtuelles globales (indésirables) et « fardeaux » potentiels (bouches inutiles) qui peuvent de ce fait être traitées par anticipation. Les camps constituent ainsi un appareil de capture et de traduction ${ }^{7}$. Ils partici-

Nations, Cultures and the Allure of Race, Londres, Allen Lane, The Penguin Press, 2000; Rahola F., «La forme-camp. Pour une généalogie des lieux de transit et d'internement du présent ", Cultures E Conflits, 68-4, 2007, pp. 31-50, et Diken B. et Laustsen C. B., The Culture of Exception: Sociology facing the Camp, London, Routledge, 2006.

3. Fumagalli V., Paysages de la peur. L'homme et la nature au Moyen Âge, Bruxelles, Éditions de l'Université de Bruxelles, 2009 (1994).

4. Delumeau J., La Peur en Occident (XIVe-XVIIIe siècles). Une cité assiégée, Paris, Fayard, 1978.

5. Giorgio Agamben évoque, en reprenant l'analyse de Carl Schmitt, les «nouvelles catégories biopolitiques » sous l'action desquelles « tous les concepts juridiques s'indéterminent ». Agamben G., Homo sacer I. Le pouvoir souverain et la vie nue, Paris, Seuil, 1985 p. 185. Danièle Lochak arrive à la même conclusion à propos des politiques migratoires actuelles. Lochak D., "La place du droit dans la radicalisation de la politique d'immigration ", contribution au VIIe congrès de l'AFSP, Lille, 2002, dact., 16 p.

6. C'est le juriste Henri Plait qui théorise ces « circonstances exceptionnelles » justifiant selon lui la concentration des pouvoirs pour obtenir la victoire. Plait H., L'État de siège et la restriction des libertés individuelles dans la guerre 1914-1919. Faculté de Paris, Auxerre, Imprimerie Staub, 1920. Il est cité par Mauran H., Les camps d'internement et la surveillance des étrangers en France durant la Première Guerre mondiale (1914-1920), Thèse d'histoire, Montpellier III, 2003.

7. Je développe cette articulation dans un article à paraître, « Le camp comme anamorphose. Comment traduire la société contemporaine depuis le camp ? », Transeuropéennes. 
pent à la manifestation de la puissance souveraine de l'État et à la production des catégories de l'altérité. Ils sont un moyen à la fois de défendre les frontières extérieures (simultanément concrétisations de la limite et conjurations de la menace ${ }^{8}$ ) et d'adapter les délimitations intérieures des sociétés statonationales car ils servent d'enclave et de frontière modulables et déplaçables selon les risques désignés par l'État, les besoins de main d'oeuvre et les contours de l'autochtonie.

La construction de ces menaces et des dispositifs pour s'en protéger transcende les distinctions classiques entre temps de paix et de guerre et entre régimes libéraux et autoritaires. Elle pose cependant des questions spécifiques aux sociétés dites « ouvertes » en ce qu'elles révèlent et prolongent les lignes de faille sociales et politiques qui les traversent et génèrent des apories éthiques et juridiques. Il s'agira ici d'interroger, à partir de formes d'inscription spatiale des étrangers dans les régimes occidentaux (camps, habitats réservés, quarantaines, zones frontières et de transit, ...), ces trois motifs récurrents de menaces auxquelles sont censés se préparer les pouvoirs publics pour protéger les sociétés contemporaines 9 .

L'objectif est principalement de montrer à partir de différents territoires les variétés et les convergences dans les représentations de l'altérité menaçante, dans l'établissement des frontières physiques et symboliques et dans les dispositifs d'anticipation et/ou de luttes contre ces menaces. Je me base à la fois sur mes travaux empiriques récents sur la question des camps et des foyers d'étrangers et sur diverses recherches actuelles participant d'une archéologie globale de la forme camp ${ }^{10}$. Elle apparaît depuis le début du XXe siècle comme une réponse des régimes politiques à ces menaces en ce que le camp offre des solutions de spatialisation ${ }^{11}$. Plastique, malléable voire liquide, l'espace du camp possède des caractéristiques qui lui permettent d'être adapté à toutes

8. Les frontières contemporaines sont plus virtuelles que matérielles. Tout comme les camps, elles participent plus d'une tentative de décélération des mobilités que d'un dispositif infranchissable. Voir Tsianos V. \& Karakayali S., "Transnational Migration and the Emergence of the European Border Regime: An Ethnographic Analysis", European Journal of Social Theory, 13-3, 2010, pp. 373-387.

9. Si ces trois formes de menaces recouvrent des réalités potentiellement différentes dans leurs modalités spatio-temporelles d'effectuation, elles s'avèrent très similaires dans les usages qui en sont faits dans les discours et souvent combinées dans les dispositifs qui leur sont opposés.

10. Pour rendre compte des liens qui s'opèrent entre représentations de l'altérité et pratiques de la mise à l'écart j'utilise à la fois des éléments des romans nationaux, des thèmes récurrents des productions culturelles ou scientifiques, des documents issus des archives publiques. Je parviens ainsi à reconstituer une trame générale des modes de mise au secret des populations, dont la présence est tout à la fois niée et combattue, que j’appelle des sans trace. $C f$. Bernardot M., «À la recherche des sans trace : cultures, espaces et citoyennetés », in Galinon-Mélénec B. (ed.) L'Homme-trace, Presses du CNRS, 2011, pp. 331-347.

11. Il s'agit de «l'ensemble des gestes par lesquels la maladie, dans une société, est cernée, médicalement investie, isolée, répartie dans des régions privilégiées et closes ». Tel l'hôpital, les institutions empiriques de mise à l'écart, incarnées par l'espace et le dispositif du camp « engage(nt) un système d'options où il y va de la manière dont un groupe, pour se maintenir et se protéger, pratique les exclusions, établit les formes de l'assistance, réagit à la peur de la 
sortes de circonstances présentées comme exceptionnelles. Cette multifonctionnalité lui confère, sous différentes formes allant des plus rudimentaires aux plus sophistiquées, une place cruciale pour étudier les États, la gouvernementalité contemporaine et les modes de production ${ }^{12}$ aussi bien que l'évolution des types de risques qu'ils cherchent à conjurer.

J'évoquerai donc successivement les trois menaces et les camps qui leurs sont opposés. J'aborderai d'abord la crainte de l'invasion, devenue depuis le XIXe siècle l'un des modèles prédominants d'appréhension des migrations, et les formes de camps qui ont servi et servent encore, combinés à d'autres moyens de contrôle, à ralentir l'entrée et la dissémination des populations étrangères. Puis, j'étudierai l'inquiétude de la subversion, caractéristique des problématiques de maintien de l'ordre et de défense de la sécurité intérieure depuis la même période. Dans ce cadre le camp est essentiellement le laboratoire policier où s'appliquent les principes de la guerre sociale et psychologique contre un ennemi de l'intérieur qui s'incarne souvent sous les traits d'un étranger ou de ses descendants. Enfin, j'évoquerai l'appréhension de la contamination, source multiséculaire de terreur collective, mais qui est devenue avec la biopolitique et l'hygiénisme un élément primordial des politiques de constitution des États et de défense des sociétés. Là encore le camp dans sa variante de sas de quarantaine trouve des localisations et des applications diverses dans le contexte de la globalisation des risques sanitaires.

\section{L'invasion}

À l'invasion, notion revisitée par l'histoire moderne et participant à l'élaboration des traditions et des romans nationaux, les sociétés contemporaines ont opposé des types de lieux de confinement destinés à contenir ou à refouler des étrangers et des migrants.

\section{Invasions, migrations et paniques morales}

L'invasion est un motif récurrent de la perception des migrations depuis le XIXe siècle. Il décrit le risque de voir les pays du Nord envahis par des populations pauvres venues du Sud. Si le thème de l'invasion est d'abord de nature militaire il a pris dans le cadre des migrations contemporaines une connotation plus civile et insidieuse. L'arrivée de «boat people » en grand nombre sur les côtes d'un pays d'Europe provoquerait le chaos et l'écroulement du sys-

mort, refoule ou soulage la misère, intervient dans les maladies ou les laisse à leur cours naturel. » Foucault M., Naissance de la clinique. Une archéologie du regard médical, Paris, PUF, 1963 , p. 14.

12. Je m'inscris ici à la suite des travaux de sociologie historique du politique, de Charles Tilly notamment. Cf. Tilly C., Contrainte et capital dans la formation de l'Europe, 990-1990, Paris, Aubier, 1992 et "War Making and State Making as Organized Crime", in Evans P. B., Rueschemeyer D. and Skocpol T. (eds.), Bringing the State Back In, Cambridge, Cambridge University Press, 1985, pp. 169-187. 
tème occidental déclinant. Ce type de schéma a été maintes fois mis en récit 13 . Un tel motif repose en partie sur des représentations structurées dans les imaginaires et les consciences collectifs à partir de constructions historiques du XIXe siècle. La crainte d'un déclin démographique de l'Occident face à une fécondité supposée incontrôlable des pays d'Asie ou du Sud a favorisé l'idée que ces derniers pourraient conquérir l'Occident riche en supplantant les populations locales par des migrants en grand nombre. « La transformation de l'émigration en colonisation et la perception de l'immigration comme invasion sont récentes. [...] Le ralentissement de la croissance de la population française, à partir du milieu du XIXe siècle, et le tarissement de l'émigration qui en est la conséquence, sont peut être responsables du rapprochement entre colonisation et émigration 14 ». On retrouve ce schéma dans les années 1930, puis dans les années 1960 formulé par les leaders d'extrême droite et plus récemment par des courants conservateurs dépeignant un chaos provoqué par l'affrontement entre locaux et envahisseurs. L'immigration de populations africaines, méditerranéennes, caribéennes, hispaniques ou asiatiques, couplée à leur installation et à la naissance d'enfants de "seconde génération ", serait la nouvelle forme que prendrait l'invasion de l'Occident, concrétisant les craintes et les alarmes. Non seulement ces groupes nouvellement installés menaceraient le caractère fantasmé comme ethniquement pur des pays d'accueil mais ils pourraient éventuellement devenir des « cinquièmes colonnes » d'une invasion militaire. Les crises pétrolières dans les années 1970 ont été instrumentalisées dans ce sens par les diffuseurs intellectuels et médiatiques de ce fantasme. La structuration de l'islamisme politique depuis les années 1980 a aussi conféré une dimension religieuse à cette crainte. Depuis les années 1990 l'inquiétude liée au réchauffement climatique a donné une nouvelle forme à cette représentation de l'invasion-migration cette fois portée par des experts et des institutions internationales. Les prévisions d'élévation du niveau de la mer et de désertification au cours du siècle à venir ont généré un important corpus quant aux risques de «mise en mouvement » de contingents de populations forcées à migrer car fuyant la dégradation voire la disparition de leurs œcoumènes et tentant coûte que coûte de se réfugier clandestinement en Occident ${ }^{15}$.

Ces différentes représentations du risque d'invasion ne s'annulent pas mais se recombinent entre elles. C'est le cas par exemple avec le thème de l'invasion de l'Australie «blanche » par des réfugiés d'Asie du Sud Est ${ }^{16}$. Des peurs collectives plus anciennes liées à la conquête coloniale du continent, son

13. Voir par exemple Moura J.-M., «Littérature et idéologie de la migration : "Le camps des Saints" de Jean Raspail ", Revue européenne des migrations internationales, 4-3, 1988, pp. 115-124.

14. Le Bras H., Le sol et le sang, La Tour d'Aigues, Éditions de l'aube, 1994, pp. 15 et 24.

15. Hein H., The Myth of Invasion. Irregular Migration from West Africa to the Maghreb and the European Union, International Migration Institute, University of Oxford, 2007.

16. Jupp J., From White Australia to Woomera. The Story of Australia Immigration, Cambridge, Cambridge University Press, 2002. 
histoire migratoire depuis le XVII ${ }^{\mathrm{e}}$ siècle et les conflits du XXe siècle ont été en fait réactivées ${ }^{17}$. «La peur d'une invasion extérieure a toujours opéré parallèlement à la culpabilité de l'occupation fondatrice de la terre en Australie. [...] Le mythe de la terre vierge et le fantasme d'une “invasion asiatique” sont les deux aspects constitutifs du nationalisme australien. Combinés, ils ont produit une profonde anxiété de l'espace et de la mobilité 18 ».

Dans le cas de la France cette idée d'invasion par des populations du Sud est aussi à replacer dans une histoire longue. L'histoire enseignée a mis en scène les exploits supposés de Charles Martel. Mais elle ne relève jamais l'installation de populations méditerranéennes. Comme le rappelle Bruno Etienne « les musulmans sont arrêtés à Poitiers en octobre 732, date très incertaine. Arrêtés ne signifie pas qu'ils sont repartis ; de nombreuses chroniques nous laissent à penser que certains s'installèrent au nord de la Loire et que la plupart restèrent et firent souche en Aquitaine 19 ». La présence sarrasine en Provence au VIII siècle a aussi été très structurante. C'est-ce que confirme Jean-Pierre Poly à propos de l'importance donnée par Marc Bloch dans La Société féodale aux "dévastations des envahisseurs sarrazins » : "La terreur barbaresque a laissé, dans le bassin méditerranéen, bien des souvenirs inauthentiques, d'autant plus nombreux que furent tôt confondus païens et sarrazins, monuments antiques et constructions mauresques. [...] l'accumulation de la peur dans la conscience collective a fait du Maure ravisseur une ombre plus grande que le corps $20 »$. Cela a des conséquences dans la présentation de la Méditerranée comme une frontière entre civilisations antagoniques et non comme un ensemble cohérent et un espace de passage et d'échange. Ces thèmes ont été réimportés dans les débats européens depuis les années 1960.

\section{Les dispositifs d'enfermement de masse contre les migrations/invasions}

Le camp a été, depuis ses premières formes contemporaines occidentales, l'un des moyens à la fois militaire et policier de lutter contre ces supposées menaces d'invasion. Les premières présences de populations issues des territoires coloniaux, importées le plus souvent par la force en Occident, ont été traitées principalement par cette technique d'installation sous contrôle 21. C'est le cas lors de la première guerre mondiale où les tirailleurs et les travailleurs indigènes coloniaux mobilisés dans le cadre de l'effort de guerre ont été pour la plupart placés dans divers camps, que ce soit sous la forme de centres d'hivernage pour les soldats noirs, maghrébins, indiens ou chinois ou de

17. Anderson W., The Cultivation of Whiteness. Science, Health and Racial Destiny in Australia, New York, Basic Books, 2003.

18. Papastergiadis N., "The Invasion Complex in Australian Political Culture", Thesis Eleven, 78, 2004, pp. 6-27, extrait p. 8. (La traduction des extraits en anglais est de l'auteur).

19. Etienne B., La France et l'islam, Paris, Hachette, 1989, p. 140.

20. Poly J.-P., La Provence et la société féodale (879-1166). Contribution à l'étude des structures dites féodales dans le Midi, Paris, Bordas, 1976, p. 4.

21. Bernardot M., Camps d'étrangers, op.cit. 
dépôts de travail pour les groupements de travailleurs indigènes. Ces camps permettaient d'une part de contrôler et de surveiller les indigènes durant leur période d'utilité stratégique ou économique et d'autre part de faciliter leur rapatriement vers leurs régions d'origine à l'issue du conflit. Le confinement spatial visait à éviter les relations avec les populations locales et notamment avec les femmes «blanches", mais aussi à ralentir la possible acculturation de ces soldats et ouvriers évitant ainsi des soucis lors de leur rapatriement.

Cette expérience initiale des migrations d'indigènes " requis " vers l'Europe a servi de base pratique et conceptuelle à l'organisation des migrations de travailleurs coloniaux durant l'entre-deux-guerres jusque dans les années 1960 sous des formes plus ou moins sévères. On la retrouve jusque dans les modalités contemporaines de traitement des migrants de passage et des demandeurs d'asile. Elles s'inscrivent elles aussi dans l'histoire longue du $\mathrm{XX}^{\mathrm{e}}$ siècle. Tout en déployant des formes d'accueil humanitaires des réfugiés, les pays occidentaux ont souvent eu simultanément une perception menaçante de l'arrivée de populations fuyant un pays. Il est vrai que ce type de déplacement de masse a parfois été instrumentalisé par les pays de départ comme l'a noté $\mathrm{H}$. Arendt à propos du régime nazi cherchant à la fois à se débarrasser de groupes «d'asociaux » et à déstabiliser ses voisins ${ }^{22}$.

Cependant, quel que soit le contexte, les lieux mis à disposition de réfugiés ont jusqu'à nos jours ressorti de la forme-camp délivrant un message allant de l'hospitalité pour certaines nationalités - dans le cas de la France les Hongrois dans les années 1950 ou les Chiliens dans les années 1970 - à la plus franche hostilité - on pense ici aux Arméniens dans les années 1920, aux Juifs allemands et autrichiens dans les années 1930 et aux Espagnols de la Retirada ou aux Franco-indochinois et aux Harkis après 1954 et 1962. Pour tous, la solution du camp a été retenue, présentée faussement comme une réponse dans l'urgence alors qu'elle était pensée et préparée par les autorités comme la moins onéreuse et la plus à même d'éviter la dispersion invasive d'étrangers considérés, selon l'expression officielle, comme «en surnombre dans l'économie nationale ${ }^{23}$ ». Car la dimension économique est essentielle dans cette mise en scène. Les étrangers sont décrits tels un fardeau pour la collectivité qui les accueille et exerçant une concurrence pour les emplois avec les nationaux. Le camp offre en cela une possibilité de mettre au travail ces hôtes indélicats dans des tâches subalternes, y compris lorsqu'il s'agit de citoyens, mais dont l'origine ou le métissage ravalent à un statut d'inférieur.

L'enfermement dans des camps facilite aussi la surveillance. Ces campscorridors se présentent alors en enclaves tournées vers l'extérieur du territoire national. Plusieurs épisodes dans les dernières décennies ont montré la conti-

22. Arendt H., Les origines du totalitarisme, tome 2, Paris, Fayard, 1982, pp. 242-243.

23. La loi française du 27 septembre 1940 s’intitule « Loi sur la situation des étrangers en surnombre dans l'économie nationale ». 
nuité des représentations et des pratiques, voire des lieux de mise à l'écart, en France comme dans d'autres pays. En France deux débarquements de Kurdes en 200124 et en 201025 ont rappelé la prégnance de cette perception de l'immigration/invasion en dépit des faibles contingents concernés (900 dans le premier cas, 130 dans le second) cependant amplifiés artificiellement par les raisonnements auto-explicatifs du « seuil de tolérance », de « l'abcès de fixation » et de « l'appel d'air ». En 2000, dans des circonstances similaires, un bateau, le Tampa Bay, chargé de réfugiés chinois tentant d'accoster en Australie, a entraîné le développement de la politique dite du Mandatory Detention (détention obligatoire) permettant l'enfermement de toute personne débarquant sans autorisation 26. Autre exemple en résonance avec l'actualité récente : le cas de réfugiés d'Haïti aux États-Unis dans les années 1980. Le premier usage de camps pour des civils sur la base américaine de Guantanamo a concerné les «boat people » haïtiens tentant d'accoster en Floride et faisant craindre un déferlement aux autorités américaines ${ }^{27}$. Le modèle du camp contre les invasions/migrations peut être réactivé à toute occasion. Lorsqu'au début des années 1990 les autorités françaises s'attendaient à l'arrivée de réfugiés algériens fuyant l'arrivée au pouvoir des islamistes en Algérie, elles ont demandé aux préfets de la zone sud de mettre en place un plan d'accueil dans les espaces militaires dont beaucoup avaient déjà servi de camps de civils dans le passé. Ce contrôle militaire des frontières et de l'espace national par le camp s'apparente à une prophylaxie sociale ${ }^{28}$ censée protéger un système social et politique présenté comme fragile parce que libéral et ainsi exposé à des menaces multiples de subversion.

\section{La subversion}

La subversion est un second modèle de perception des mobilités et de la présence d'étrangers. Plus que la prophétie invasive, catastrophe possible mais rare, l'élément subversif, agent d'une invasion lente, est omniprésent à force d'être potentiel et indétectable. Le camp d'internement permet d'anticiper cette menace invisible en traitant les civils et les groupes minorisés en suspects avant leur éventuel passage à l'acte.

24. Mohseni C., «L'accueil des demandeurs d'asile en France : le cas des Kurdes de l'East Sea », Hommes et migrations, 1250,2002 , pp. 59-64.

25. «Une centaine de réfugiés retrouvés sur une plage en Corse », Le Monde.fr, 22 janvier 2010, http://.lemonde.fr/societe/article/2010/01/22.

26. Papastergiadis N., op. cit.

27. Simon J., "Refugees in a Carceral Age: The Rebirth of Immigration Prisons in the United States", Public Culture, 10-3, 1989, pp. 577-607.

28. Inda J. X., "Border Prophylaxis : Technology, Illegality, and the Government of Immigration”, Cultural Dynamics, 18-2, 2006, pp. 115-138. 
La subversion structure la perception par les autorités et les élites des migrations et de la présence d'étrangers dans les territoires occidentaux. Cette menace est en effet souvent décrite comme permanente mais sourde et difficilement détectable. Tout comme la crainte de l'invasion avec qui elle peut se combiner, elle s'autoalimente en raison de sa difficulté à être confirmée dans les faits. Déjà très présente dans les imaginaires et les discours au XIXe siècle à propos des révolutionnaires européens puis des groupes anarchistes, elle est conçue comme l'intervention invisible et illégale de petits groupes d'activistes, qui telles des sociétés secrètes, œuvrent contre la sécurité de l'État. Au-delà de cette dimension idéologique, la subversion apparaît comme étant potentiellement manipulée par des gouvernements étrangers voire des organisations mondialisées. C'est un des thèmes antisémites classiques. À ce caractère actif de la subversion s'ajoute une dimension plus passive qui fait de la présence d'étrangers une menace pour les mœurs et l'éducation, la culture et la langue, l'identité ou la virilité nationales 29.

C'est au milieu du XIXe siècle, dans le cadre de l'invention des polices modernes et de la préparation des guerres à venir, que les gouvernements européens réfléchissent à la possibilité de mettre hors d'état de nuire des ressortissants étrangers. Les premières listes systématiques les identifiant sont constituées afin de les arrêter, de les expulser ou de les interner. La suspicion à leur endroit peut s'étendre aux nationaux d'origine étrangère ou aux parias nationaux. Durant la première guerre mondiale les citoyens de nationalité allemande ou autrichienne notamment, sont considérés comme des ennemis potentiels dans la plupart des pays occidentaux en guerre avec l'Allemagne. Ils font l'objet d'une surveillance policière et parfois de manifestations de xénophobie populaire. Les populations mobiles ${ }^{30}$ sont aussi suspectées de pouvoir, du fait de leurs déplacements, renseigner l'ennemi sur la position des troupes françaises.

La question de la loyauté des double-nationaux (les Anglo-saxons parlent de citoyens en pointillés [hyphenated citizenship]) occupe une place prépondérante dans l'histoire contemporaine de la subversion. Elle est tout à fait déterminante dans la politique américaine et canadienne vis-à-vis, entre autres, des nippo-américains et de germano-américains durant la Seconde Guerre mondiale qui conduit à l'arrestation, à la spoliation, à la déportation et à l'internement de centaines de milliers de personnes. À ce propos Mike Davis évoque les procédés répétitifs de construction de la menace japonaise dans les romans repris par le cinéma nord-américain dès le début du XXe siècle: «Dans

29. Mosse G. L., L'Image de l'homme. L'invention de la virilité moderne, Paris, Éditions Abbeville, 1997.

30. Thomas H., La production des exclus. Politiques sociales et processus de désocialisation sociopolitique, Paris, PUF, 1997. 
cette sinistre généalogie, il est fort surprenant que la fiction de la destruction de Los Angeles ait été inaugurée en 1909, non par le tremblement de terre, l'inondation ou l'incendie mais par l'invasion de la Californie du Sud. [...] Avec une précision d'horloge une armée japonaise clandestine, préventivement déguisée en humbles ouvriers, en personnels de maison et travailleurs agricoles, attaquent les principales cités californiennes. San Francisco se rend rapidement devant les hordes de "Bridés" 31 ».

Cette question reste de nos jours encore très prégnante dans l'appréhension des citoyens et des migrants selon leurs origines et les conflits. L'idée de subversion laisse libre cours au traitement de tel groupe ou individu en tant que suspect. Ainsi réifié ou catégorisé l'à demi national devient un danger et un possible ennemi de l'intérieur. Aucune distinction de classe, de genre, d'âge ou de race n'invalide ce raisonnement et chacune peut le renforcer ${ }^{32}$. L'enfant d'étranger peut ainsi être un ennemi en devenir, l'homme un soldat, la femme une séductrice, l'ouvrier un saboteur, l'intellectuel un agent. Les très nombreuses productions culturelles qui mettent en scène les modalités de lutte contre le terrorisme - dans le cadre de la guerre contre la terreur notamment participent de ce processus d'accréditation de la menace subversive et de légitimation des violences et des atteintes aux droits, y compris quand elles dénoncent des comportements institutionnels ${ }^{33}$.

Ces associations sont régulièrement réactivées depuis les conflits de la décolonisation en Europe lorsque les migrants post-coloniaux sont aussi d'anciens indigènes révoltés. Elles démontrent par ailleurs une transitivité forte avec les catégories de la criminalité organisée et du terrorisme et imprègnent les postures hostiles vis-à-vis des migrants légalement rendus clandestins et, au-delà, les attitudes défiantes à l'encontre de leurs descendants. Redoublant et renforçant, en les légitimant, les discriminations dont sont déjà victimes ces groupes, les politiques répressives à leur encontre sont, depuis les attentats des années 2000, de plus en plus sévères et de moins en moins officieuses. L'anthropologue Nadine Naber appelle cela un « internement psychique » [internment of the psyche] et le sociologue Martin French parle de "l'ombre du camp » qui pèse sur les minorités ${ }^{34}$. Des citoyens double-nationaux de confession musulmane deviennent collectivement la cible d'une surveillance et d'une suspicion accrues et peuvent être individuellement la cible d'exfiltrations violentes ${ }^{35}$.

31. Davis M., Ecology of Fear. Los Angeles and the Imagination of Disaster, New York, Metropolitan Books, 1998, pp. 293-295.

32. Linn N., Lea S., "A Phantom menace and the new apartheid: the social construction of asylum-seekers in the United Kingdom”, Discourse E Society, 14.4, 2003, pp. 425-452.

33. Erickson C.W., “Counter-Terror Culture: Ambiguity, Subversion, or Legitimization?”, Security Dialogue, 38-2, 2007, pp. 197-204.

34. Naber N., "The Rules of Forced Engagement. Race, Gender, and the Culture of Fear among Arab Immigrants in San Francisco Post-9/11”, Cultural Dynamics, 18-3, 2006, pp. 235-267; M. French, "In the Shadow of Canada's Camps", Social E Legal Studies, 16-1, 2007, pp. 4969. 
Le camp est, depuis plus d'un siècle, un dispositif central dans la lutte des États occidentaux contre la subversion à la fois dans les espaces coloniaux et dans les territoires métropolitains. Dans les premiers, s'il s'agit d'un outil de contrôle et de mise en valeur du territoire, il fonctionne simultanément comme un moyen de lutte contre les révoltes éventuelles et la circulation incontrôlée des indigènes et en tant que moyen de séparation de ceux-ci d'avec les colons. Il peut être mis en œuvre de manière très rudimentaire dans les formules du « resserrement » et du cantonnement qui s'avèrent souvent n'être qu'un refoulement brutal de groupes renégats vers des zones hostiles ou au moins non exploitables, en Algérie ou en Australie par exemple.

Ensuite, depuis l'espace de la plantation pour l'exploitation des esclaves dans les Caraibes et en Louisiane notamment, les camps sont des organisations rationnelles de mise au travail 36 et d'anticipation du potentiel subversif, au sens tant politique que racial, de ces concentrations humaines. Les camps nègres, selon leurs promoteurs souvent issus des Lumières, permettent de surveiller, d'appliquer la terreur du système esclavagiste et de réguler les relations inter-raciales ${ }^{37}$. Enfin c'est dans le cadre des guerres coloniales de la fin du XIXe siècle que le camp acquiert sa dimension proprement politique ${ }^{38}$ et même sa date de naissance dans l'opinion publique internationale sous le nom de campo de reconcentracion. Il devient alors le moyen principal de lutte contre les mobilisations indigènes et la subversion indépendantiste et anti-coloniale en étant utilisé sciemment contre des civils afin d'éviter qu'ils protègent et ravitaillent des insurgés 39 .

Dans l'espace métropolitain, si l'on excepte les expériences de concentration (associées à des déportations) de pauvres, mendiants, et autres "Bohémiens », depuis longtemps associés « à la crainte du renversement des hiérarchies 40 ", les « colonies " pour enfants, qui se développent à partir des années 1830, constituent une modalité de camp combinant redressement, punition et expulsion du territoire d'éléments asociaux construits comme subversifs. Comme le montre Ann Stoler ces établissements constituent «un

35. Stasiulis D., Ross D., "Security, Flexible Sovereignty, and the Perils of Multiple Citizenship", Citizenship Studies, 10.3, 2006, pp. 329-348.

36. Hordèj P. (ed.), L'Esclave et les plantations. De l'établissement de la servitude à son abolition, Rennes, PUR, 2008.

37. Dawdy S. L., "Proper Caresses and Prudent Distance: A How-to Manuel from Colonial Louisiana", in Stoler A. L. (ed.), Haunted by Empire. Geography of Intima in North American History, Durham, London, Duke University Press, 2006, pp. 140-162.

38. Voir par exemple pour les Philippines et la Namibie: Mc Coy A. W., Policing America's Empire. The U.S., the Philippines and the Rise of the Surveillance State, Madison, University of Wisconsin Press, 2009; Hull I. U., Absolute Destruction, Military Culture and the Practices of War in Imperial Germany, Ithaca, London, Cornell University Press, 2005.

39. Le Cour Grandmaison O., De l'indigénat. Anatomie d'un monstre juridique : le droit colonial en Algérie et dans l'empire français, Paris, La Découverte/Zones, 2010.

40. Delumeau J., op. cit., p. 189. 
archipel carcéral de l'empire qui reliait les colonies pénales aux coopératives agricoles et aux maisons de redressement. Cela connectait les stratégies de confinement de la métropole aux colonies à travers le monde impérial. [...] Les orphelins, les jeunes urbains abandonnés et les dissidents politiques devaient être formés et recrutés pour s'établir dans les étendues dilatées de l'empire ${ }^{41}$ ».

Mais c'est surtout avec la Première Guerre mondiale que le camp devient l'espace central de la lutte contre la subversion. L'ensemble des pays belligérants ont recours à l'internement administratif des ressortissants étrangers afin d'éviter leurs éventuelles activités subversives ${ }^{42}$. À ces objectifs militaires sont superposées des fins policières qui se développeront durant l'entre-deuxguerres. S'élaborent et s'expérimentent dans les camps les techniques de guerre psychologique et sociale. Ce dispositif spatial et politique se développe jusqu'aux conflits asymétriques actuels ${ }^{43}$ en passant par les guerres d'Algérie ou d'Irlande, érigées en modèles ${ }^{44}$. S'y systématisent les dispositifs permettant de différencier les éléments subversifs de la masse des internés. Si le procédé même de la concentration expose les autorités gestionnaires à des expressions politiques des ensembles ainsi artificiellement constitués, cela participe in fine de la volonté d'identifier des "meneurs » qui peuvent être isolés et réprimés. Le camp est pensé comme un laboratoire ${ }^{45}$. L'internement s'impose non plus comme une techné répressive occasionnelle dans le cadre d'un recours éventuel de l'État de droit à l'arbitraire mais comme un moyen routinisé de lutte contre toutes formes de subversion. On le retrouve dans les situations de crises insurrectionnelles, de guerres civiles, de sorties de conflits armés, de prises de contrôle d'un territoire ou d'un appareil gouvernemental, de luttes contre un ennemi civil intérieur, de guerres de décolonisation, etc. Par ailleurs une passerelle peut être faite entre ces guerres à distance et les luttes contre les ennemis de l'intérieur en particulier les guerres contre la criminalité qui se résument souvent à une répression des minorités ${ }^{46}$.

41. Stoler A. L., Along the Archival Grain. Epistemic Anxieties and Colonial Common Sense, Princeton, Princeton University Press, 2009, p. 131.

42. Stibbe M., "The Internment of Civilians Belligerents States during the First World War", Journal of Contemporary History, 41, 2006, pp. 5-19.

43. Welch M., "Detained in occupied Iraq. Deciphering the narratives for neo-colonial internment", Punishment \& Society, 12, 2010, pp. 123-147.

44. MacMaster N., "Torture : from Algiers to Abu Ghraib”, Race E Class, 46-2, 2004, pp. 1-21; Kennedy-Pipe C., Mumford A., "Torture, Rights, Rules and Wars: Ireland to Irak", International relations, 21-1, 2007, pp. 119-126.

45. Bernardot M., «Le garde et l'interné : Essentialisation des catégories et subversion des clivages dans les centres d'internement français de la guerre d'Algérie (1959-1962) », Travailler, Revue internationale de psychopathologie et de psychodynamique du travail, 16, 2006, pp. 8196.

46. Gilmore R.W., Golden Gulag. Prisons, Surplus, Crisis, and Opposition in Globalizing California, Berkeley, University of California Press, 2007; Rigouste M., "L'ennemi intérieur, de la guerre coloniale au contrôle sécuritaire », Cultures E Conflits, 67, 2008, pp. 157-174. 
Cette visée politique est parfois articulée à des objectifs d'hébergement de refugiés qui peuvent abriter des opposants ou des criminels présumés. C'est par exemple le cas lors de l'accueil des Espagnols républicains ou des migrants et exilés italiens suspectés d'être communistes en France à la fin des années 1930. Les autorités, ainsi que la presse et les élites plus généralement, décelaient derrière le migrant un agent idéologique doublement menaçant par ses fins énoncées en tant que subversives et sa mobilité hors de contrôle. Devenu une forme globale dans toutes ses occurrences, le camp est maintenant l'outil central de la gestion des migrations, de la force de travail contrainte et des déplacements humains de masse partout associées au risque subversif ou terroriste et à la criminalité organisée. La détention arbitraire permet dorénavant de combattre à la fois les revendications économiques ou citoyennes des migrants illégalisés et leurs mobilisations contre leurs conditions de vie, d'internement ou de déportation.

\section{La contamination}

Dans les représentations collectives, la contamination est souvent associée à l'influence négative d'éléments étrangers. Les camps déployés pour faire face à cette menace associent les dimensions d'espace de quarantaine et de gestion des migrations.

\section{Du germe étranger à l'étranger contaminant}

La crainte de la contamination est, tout comme l'invasion et la subversion, elle aussi ancienne. Elle est présente depuis les épidémies antiques et médiévales qui ont structuré les représentations de la maladie et généré les grandes peurs qui agitent les sociétés. Leurs craintes ont inspiré de nombreuses œuvres du Panthéon littéraire dont les schémas sont encore très présents en particulier dans la production cinématographique contemporaine mêlant des situations de chaos politiques, d'invasions et de confinements des malades. Les épidémies ont eu un rôle central dans la structuration des États depuis le XVIIe siècle ${ }^{47}$. Avec le développement de la médecine expérimentale, les autorités ont mis en place des politiques sanitaires et prophylactiques appliquant un hygiénisme coercitif aux voyageurs, aux étrangers et aux indigènes. La présence d'étrangers est historiquement associée dans les discours aux risques d'épidémies. C'est le cas par exemple dans les années 1920 à Paris et en région parisienne concernant les populations kabyles qui y résident. Pour partie soldats démobilisés après la Grande guerre, ces ouvriers sont décrits comme vivants dans de déplorables conditions d'hygiène et susceptibles de répandre des maladies, notamment la tuberculose et la syphilis, endémies souvent combinées à des représentations raciales et morales des indigènes débouchant sur

47. Foucault M., Sécurité, territoire, population, Cours au Collège de France, 1977-1979, Paris, Gallimard, Seuil, 2004. Voir en particulier leçons du 29 mars et du 4 avril 1978, pp. 319-370. 
l'idée d'une impossible intégration. Les premières politiques sociales en direction de ces publics comporteront des volets sanitaires et policiers spécifiques et articulés ${ }^{48}$. Il est fréquent que l'arrivée de migrants ou de réfugiés déclenche des craintes sanitaires dans les pays d'accueil ou de passage. Les migrations contemporaines sont rapprochées de la prostitution et des risques de transmissions de maladies « honteuses ». Avec l'accroissement de la présence de migrants africains en Europe se sont aussi diffusées les inquiétudes concernant les pathologies « exotiques ». La rumeur joue un rôle important dans la diffusion de ces stéréotypes en lien avec les stratégies de communication des autorités.

Des procédures de regroupement et de mises à l'écart pour vérifier l'absence de maladies ont été appliquées depuis la fin du XIXe siècle aux migrants nouvellement venus. Les dépôts d'étrangers installés aux frontières et dans les ports d'alors ont fourni le cadre d'une sélection et d'un diagnostic sommaires visant à ségréger puis à renvoyer les «éléments » porteurs de maladies et traités comme agents pathogènes. Dans les années 1920 les réfugiés arméniens arrivés à Marseille ont été parqués. Un courrier préfectoral raconte les modalités de leur prise en charge : « La semaine dernière a vu débarquer à Marseille quinze cent de ces émigrants qui ont subi les mesures prophylactiques maritimes habituelles et de salubrité publique. Ce service est assuré par la police spéciale des ports et la santé maritime. C'est ainsi qu'au Frioul eurent lieu les opérations régulières de vaccination, désinfection, épouillage, visa des passeports, délivrance des passeports sanitaires 49 ». Cette détection ne se limitait pas à l'arrivée mais précédait bien souvent le départ des travailleurs coloniaux organisé par des employeurs. Dans les centres d'embauche avant le voyage vers le pays d'accueil les recruteurs évaluaient la force et l'état de santé des candidats. La sélection a porté aussi sur les maladies mentales ou les déficiences physiques. À Ellis Island aux États-Unis ou dans les ports australiens, les personnes souffrant de problèmes mentaux étaient interdites de territoire et refoulées. Il s'agit ici d'un point qui permet de relier question migratoire, problématique sanitaire et modèle du camp dans une perspective de longue durée. C'est tout particulièrement le cas en Australie. Comme le montrent Alison Bashford et Carolyn Strange « les politiques de détention actuelles des demandeurs d'asile arrivant par bateau en Australie sont annoncées par l'histoire de la quarantaine et des politiques d'internement des civils ennemis en temps de guerre.[...] Les pratiques carcérales actuelles imposées aux réfugiés reproduisent d'anciennes formes de détention obligatoire dans lesquelles l'altérité, le statut d'ennemi, la dangerosité, l'indésirabilité sont regroupées 50 ».

48. Bernardot M., Loger les immigrés. La Sonacotra 1956-2006, Bellecombe-en-Bauges, Editions du Croquant, 2008.

49. Courrier de l'inspecteur général au préfet des Bouches-du-Rhône, 10 octobre 1923, ADBdR, 4 M 1195.

50. Bashford A., Strange C., "Asylum-Seekers and National Histories of Detention", Australian Journal of Politics and History, 48-4, 2002, pp. 509-527. 
L'indigent comme l'indigène sont aussi interdits de séjour car ce pouvoir de détention est appliqué différemment selon les races. A. Bashford et C. Strange citent un dirigeant du département de santé publique du Commonwealth expliquant en 1928 que « pour les Chinois, les Kanaks et les Aborigènes, l'isolement sous un strict contrôle est la seule chose que l'on puisse envisager. Pour les Européens indigents ou débiles, un contrôle identique est nécessaire 51 ». Quarantaine et détention arbitraire sont combinées dans une technologie par laquelle «l'Australie blanche » a été mise en œuvre. Les législations ont œuvré à la création d'une Australie imaginaire " propre, pure, immunisée, non contaminée 52 ». Ainsi la détention légale de non-criminels, les dispositifs de quarantaine et la gestion de l'immigration sont regroupés dans la problématique centrale de la sécurité et de la défense nationale plus ou moins explicitement racialisée et traduite dans des termes militaires. La contamination de l'Australie blanche par la maladie a de la sorte été assimilée à l'invasion par des candidats asiatiques à l'immigration.

Cette modalité de contrôle des frontières des États est toujours présente. Elle est même renforcée par l'accroissement de la circulation des marchandises et des individus. Les épisodes pandémiques de ces dernières décennies ont conduit à une élévation de la surveillance sanitaire à l'échelle internationale. Les processus de nomination qui désignent des foyers infectieux situés hors de l'Occident font des grippes des importations chinoises, asiatiques, mexicaines... Par ailleurs, ces maladies sont associées à des vecteurs animaux de transmission. Les schèmes de représentation des espèces nuisibles et invasives communiquent étroitement avec une tendance à l'animalisation dans les discours xénophobes et racistes qui prévalent dans la gestion des migrations ${ }^{53}$. Par ce biais, un lien est instauré entre le « germe étranger » et « l'étranger prohibé 54 ».

\section{Le camp est à la fois solution de quarantaine et source d'inquiétude sanitaire}

Les camps d'étrangers s'inscrivent pour une part dans la tradition du lazaret et de la léproserie, et pour une autre dans celle de la quarantaine maritime. Ils en sont la forme moderne dans la mesure où ils en partagent la fonction préventive en matière sanitaire mais en l'étendant de manière répressive, comme vu précédemment, à d'autres types de risques, notamment démographiques et politiques. Cette finalité est le plus souvent articulée à d'autres, pour la gestion des crises et des migrations. Les camps fonctionnent alors comme un sas de décontamination ou de confinement dans des circonstances exceptionnelles alors que les stations de santé (qui ont remplacé les quaran-

51. Idem, p. 515

52. Ibidem, p. 515

53. Coates P., American Perceptions of Immigrants and Invasive Species. Strangers on the Land, Berkeley, University of California Press, 2006.

54. Bashford A., Strange C., op. cit., p.514 
taines et les lazarets) sont intégrées aux systèmes de santé et de protection des frontières. Cependant la surveillance des étrangers et la suspension des libertés de communication et d'aller et venir se retrouvent dans les deux espaces. Les camps sont une sorte de quarantaine généralisée qui permet la sélection des individus. C'est en pratique un espace de suspension des libertés, des droits et du temps, le domaine de l'indéfini. Dans le contexte actuel de contrôle de la mobilité, la désignation de pays «à risques », non pas pour leurs habitants mais pour les pays d'accueil éventuels, rapprochent les logiques sanitaires, les principes de la lutte contre les migrations d'indésirables et la gestion des «populations à risques » dans les politiques publiques des pays dont le Welfare s'est transformé en Workfare et en Warfare.

Un autre point permet d'opérer un rapprochement entre la quarantaine, l'internement et le confinement migratoire. Les camps sont à la fois des lieux de répression et de politiques sanitaires, non seulement lorsqu'ils sont présentés comme humanitaires accueillant des civils déplacés ou sinistrés mais aussi lorsqu'il s'agit de leur appliquer une contention collective et sans limite de temps. C'est ce que montre l'exemple du centre d'assignation à résidence surveillé (CARS) de Larzac ouvert en 1957. Les conditions de vie et les mauvais traitements infligés aux internés entraînent une dégradation rapide de leur santé. Très vite les autorités mettent en place un service de santé pour veiller à maintenir en vie les Algériens détenus qui ne cessera de se développer pour traiter des épidémies de grippe, de tuberculose et de pathologies psychiatriques ${ }^{55}$. Durant l'année 1960 le service de santé du camp de Larzac effectuera plus de 30000 consultations pour environ 2000 internés. Le directeur du centre décrivait ainsi la situation: "L'hospitalisation des assignés à Rodez présente des difficultés (escorte et garde) qui sont lourdes budgétairement. L'idéal serait que le service de santé du centre soit apte à soigner sur place tous les malades. [...] Si le centre doit recevoir, comme il est prévu, plusieurs milliers d'assignés, une solution de "polyclinique" est à envisager, comme devant être, en fin de compte, la moins onéreuse 56 ». Dans les situations de confinement « d'indigènes coloniaux ", les représentations liant race et pathologies sont la norme. Le préfet ayant sous son autorité le centre de Larzac le confirme : «La présence des Nord-africains dans les CARS pose des problèmes relatifs à l'état sanitaire. Deux sont particulièrement importants : la prévention des maladies épidémiques et le dépistage de la tuberculose 57 ».

Par essence un camp, qu'il ait ou non des fins de destruction d'un groupe social, est un site amené à gérer une masse humaine sans moyen de subsistance autonome et dont il faut anticiper les problématiques de vie collective notam-

55. Bernardot M., «Le garde et l'interné », op. cit.

56. Courrier du directeur du CARS de Larzac au médecin lieutenant colonel d'Auch, 26 juin 1959, CAC MI 1977381 art. 12.

57. Courrier du préfet de l'Aveyron au directeur du CARS du Larzac, 10 septembre 1959, CAC MI 1977381 art. 12. 
ment en matière alimentaire, sanitaire, d'évacuation des eaux usées et de sépultures. Tous ces aspects ne manquent pas de provoquer des conflits entre les internés, entre ces derniers et leurs gardes et avec les riverains lorsqu'il y en a. Des épidémies de choléra, de typhus, de grippe ou de dysenterie peuvent se déclarer. Cela provoque un fort effet d'engagement sanitaire des autorités y compris lorsque les internés sont considérés comme des ennemis. Elles cherchent à la fois à protéger la santé collective des détenus et à éviter la propagation des épidémies à l'extérieur. Là encore on peut constater une proximité avec les problématiques de protection des personnels soignants dans les structures hospitalières en situation de pandémie. Dans un cas comme dans l'autre, internement et quarantaine nécessitent d'englober malades et personnels dans des procédures de confinement identiques. Cependant le corps malade et souffrant du détenu perd progressivement sa biolégitimité dans l'espace de la détention administrative ${ }^{58}$. Ses affections et ses souffrances ont tendance à être considérées par les autorités comme fausses et destinées à ralentir l'activité policière ou le processus de déportation.

Les récentes menaces de pandémies grippales ${ }^{59}$ ont amené de nombreux gouvernements à programmer des plans de quarantaine dans des zones sécurisées et de la possibilité de transformer certains espaces (hôpitaux, lieux contaminés, ...) en zones isolées sous contrôle militaire. L'Union européenne étudie la possibilité de se doter de moyens coordonnés d'alerte et de contrôle sur le modèle américain du Center for disease control (CDC). « La quarantaine connaît donc un regain de notoriété comme en témoignent les fiches d'informations préparées et établies par le CDC dans lesquelles on peut lire que "la quarantaine est médicalement très efficiente pour protéger le public de la maladie" 60 ». Il faut penser conjointement ces espaces et les nouveaux territoires de gestion des mobilités (zone d'attente, centre de rétention, d'accueil ou de déportation) comme autant d'outils d'une biopolitique renouvelée, non pas tant exceptionnels que latents et adaptables à toutes fins. Les menaces et les attentats bioterroristes participent aussi à accréditer l'idée d'un lien entre mobilités migratoires, guerre contre la terreur, criminalité organisée et épidémies.

\section{Accréditer la menace pour légitimer l'arbitraire}

La forme-camp est-elle une exception ? Les régimes occidentaux ont-ils eu uniquement recours à l'internement et à la mise à l'écart contrainte à l'occasion de crises militaires, politiques ou sanitaires justifiant la mise en place de

58. Fischer N., « Le corps comme champ de bataille. Politiques de l'humanitaire dans un centre de rétention français ", in Kobelinsky C., Makaremi C. (eds.), Enfermés dehors. Enquêtes sur le confinement des étrangers, Bellecombe-en-Bauges, Editions du Croquant, 2009, pp. 85103.

59. Gensinia G. F., Yacouba M. H., Contia A. A., "The concept of quarantine in history: from plague to SARS”, Journal of Infection, 49, 2004, pp. 257-261.

60. Idem, p. 260. 
politiques spécifiques visant à défendre la société ? Non l'encampement est un mode de spatialisation ancien que l'on retrouve dans les processus de production et de domination de la colonisation et du traitement des pauvres et des déviants. La période contemporaine a été marquée en revanche par une cristallisation des formes proto-internementales dans un modèle original. S'est engagée depuis la fin du XIXe siècle une institutionnalisation de l'internement, c'est-à-dire son incorporation dans les architectures légales et constitutionnelles, combinant des phases de manifestations intenses du camp avec son usage latent et permanent à l'encontre de groupes sociaux construits comme représentant une menace contre l'ordre social. En effet la politisation des questions migratoire et ethnique dans les sociétés contemporaines a favorisé la réémergence de l'association de l'invasion, la subversion et la contamination avec les migrants, les étrangers, les parias nationaux et plus largement avec les populations envisagées comme «à risque ». Dans les circonstances de la globalisation et de la post-modernité, le modèle du camp est maintenant utilisé comme un moyen de lutte tout terrain contre ces différentes menaces et démontre sa plasticité. Il interroge néanmoins les systèmes démocratiques notamment concernant l'État de droit et le recours systématique à des lois d'exception qui remettent en cause les principes fondateurs des régimes occidentaux (privation arbitraire de liberté, arrestation anticipée, rétroactivité de la loi, peines collectives et sans durées définies, zones grises de recours à une violence sans limite). L'accélération des mobilités, comme la réémergence de risques pandémiques, réamorce les tensions entre liberté de circulation, cruciale pour le marché et la démocratie, et le contrôle des frontières matérielles et symboliques, déterminant pour la protection des sociétés et des États qui les gouvernent. Dans les trois cas ici étudiés de craintes et de camps qui leurs sont opposés, on peut constater une tendance forte à l'accréditation des premières par les seconds. Ces outils de lutte contre les risques, caractéristiques des États contemporains, matérialisent en fait aux yeux des sociétés des peurs qui restent le plus souvent hypothétiques mais qui toutes partagent le fait de désigner des altérités comme causes. Cela participe de stratégies étatiques de justification de l'action publique et de renforcement des contours nationaux inventés sur lesquels elle se fonde. Le paradoxe reste pourtant que ces politiques s'attaquent en réalité aux sociétés qu'elles sont censées défendre, remettant en cause les principes même de leur légitimité souveraine par la privatisation des fonctions régaliennes dans l'exercice de la guerre et de la sécurité. 